\title{
Effects of activated carbon $N$-acetylcysteine sustained-release microcapsule on dipeptidyl peptidase IV expression in young rats with non-alcoholic fatty liver disease
}

\author{
HONGPING ZHOU $^{1 *}$, TINGTING SHI $^{2 *}, \mathrm{JUN} \mathrm{YAN}^{1}, \mathrm{XIAOJIN} \mathrm{CHEN}^{1}$, \\ LI LIAO $^{1}$, SHIYONG ZHAO ${ }^{1}$, HONGYING FANG ${ }^{2}$ and RANGXIAO ZHUANG ${ }^{2}$ \\ ${ }^{1}$ Department of Pharmacy, Hangzhou Children's Hospital, Hangzhou, Zhejiang 310014; \\ ${ }^{2}$ Department of Pharmaceutical Preparation, The Xixi Hospital of Hangzhou Affiliated to Zhejiang \\ University of Traditional Chinese Medicine, Hangzhou, Zhejiang 310023, P.R. China
}

Received September 28, 2016; Accepted June 22, 2017

DOI: $10.3892 /$ etm.2017.5128

\begin{abstract}
Non-alcoholic fatty liver disease (NAFLD) in children has become the most common liver disease influencing adolescent health and one of the most influencing chronic liver diseases among children in Chinese wealthy families, particularly in coastal regions. However, the medicine available for the treatment of NAFLD is deficient. In order to solve this problem, our team studied the activated carbon $N$-acetylcysteine (NAC) sustained-release microcapsule, which improves the oxidation resistance, bioavailability and drug stability of acetylcysteine and reduces toxic and side effects. In addition, it accords with the characteristics of medication in infants and children. The present study mainly discusses whether the activated carbon NAC sustained-release microcapsule has effects on dipeptidyl peptidase IV (DPPIV) activity and protein in young rats with NAFLD, and whether it has the effect of an DPPIV inhibitor, hoping to provide new thoughts and methods with respect of basic studies on young rats with NAFLD/non-alcoholic steatohepatitis.
\end{abstract}

\section{Introduction}

With the globalization and younger-age trend of obesity, non-alcoholic fatty liver disease (NAFLD) has become one of the most prevalent chronic liver diseases among children in developed countries and wealthy Chinese families,

Correspondence to: Professor Rangxiao Zhuang, Department of Pharmaceutical Preparation, The Xixi Hospital of Hangzhou Affiliated to Zhejiang University of Traditional Chinese Medicine, 2 Hengbu Road, Hangzhou, Zhejiang 310023, P.R. China

E-mail: zhuangrangxiao@sina.com

${ }^{*}$ Contributed equally

Key words: $N$-acetylcysteine, dipeptidyl peptidase IV, non-alcoholic fatty liver, microcapsule, activated carbon particularly in coastal regions (1-5). Dipeptidyl peptidase IV (DPPIV) is a type of transmembrane serine protease, $\mathrm{T}$ cell activation protein and adenosine deaminase binding protein, which is also known as cluster of differentiation 26. Besides the hydrolytic activity of peptidase, it also has regulating effects on fat metabolism, immunity and inflammation (6). The results of previous studies have revealed that the activity of DPPIV is associated with chronic liver diseases, including NAFLD (7-9). Previously, Machado et al (10) and Qu et al (11) effectively combined the powerful antioxidant $N$-acetylcysteine (NAC) and activated carbon for medicine with good adsorption properties and biocompatibility, and prepared an NAC-activated carbon sustained-release microcapsule (ACNAC). ACNAC reduced various side effects of NAC and enhanced the half-life of drugs and bioavailability. Based on previous studies (12-15), the present study aimed to further explore the effects of ACNAC on young rats with NAFLD by establishing a model of young rats with NAFLD in order to detect the serum DPPIV activity level and the expression of DPPIV protein in the liver. The present study discusses whether ACNAC is a DPPIV inhibitor, and whether it is able to protect young rats with NAFLD.

\section{Materials and methods}

Preparation of non-alcoholic fatty liver model and sample collection. A total of 64 healthy, clean and weaned Sprague-Dawley male rats (weight, $51.93 \pm 4.28 \mathrm{~g} ; 21$ days old) were purchased from the Animal Center of Zhejiang Academy of Medical Sciences [Hangzhou, China; animal license number: SCXK (Zhe) 2014-0001]. The rats had ad libitum access to a standard commercial diet and water, with the exception of preoperative fasting and were kept in rooms maintained at $22 \pm 1^{\circ} \mathrm{C}, 40-60 \%$ relative humidity with a $12 \mathrm{~h}$ light/dark cycle throughout the experiments. All experiments were performed in accordance with the National Institutes of Health Guide for the Care and Use of Laboratory Animals, and ethical approval was granted by the Animal Care Committee of Xixi Hospital Affiliated to Zhejiang University of Traditional Chinese Medicine (Hangzhou, China). Following 1 week of 
acclimatization to these conditions, rats were randomly divided into the following groups ( $\mathrm{n}=8$ per group): Normal group, fed standard diets; model group; fed high-fat diets (69\% basic feed, $10 \%$ lard oil, $2 \%$ cholesterol, $5 \%$ sugar, $0.5 \%$ cholate, $10 \%$ yolk powder, $3 \%$ yeast powder and $0.5 \%$ decavitamin); polyene phosphatidyl choline group; administered $60 \mathrm{mg} / \mathrm{kg}$ PPC (Sanofi-aventis; Beijing, China) by gastric perfusion daily and fed high-fat diets; NAC group, administered $60 \mathrm{mg} / \mathrm{kg}$ NAC (Whu hoyo Co., Ltd, Wuhan, Hubei) by gastric perfusion daily and fed high-fat diets; activated carbon release microcapsule group, administered $60 \mathrm{mg} / \mathrm{kg}$ activated carbon release microcapsule (Zhejiang Hangzhou Wood industry Co., Ltd., Huzhou, Zhejiang) and fed high-fat diets; and ACNAC low-, medium- and high-dose groups, fed high-fat diets and administered 15, 30 and $60 \mathrm{mg} / \mathrm{kg}$ of ACNAC [made in-house as described previously (12-15)], respectively by gastric perfusion daily. Following 7 weeks of these treatments, the young rats were sacrificed, and their blood and livers were collected and stored at $-80^{\circ} \mathrm{C}$ for future use.

Calculation of liver index. The liver-wet weight and the body weight of the young rats were calculated. The liver index was calculated according to the following formula: Liver index $(\%)=$ liver-wet weight / body weight $\mathrm{x} 100$.

Observation of the degree of fatty degeneration in the liver tissue of young rats by hematoxylin and eosin (HE) staining. Fresh liver tissues from rats of each group were treated in $4 \%$ paraformaldehyde for $24 \mathrm{~h}$ at room temperature, dehydrated with alcohol prepared according to a set gradient $(75,85$, 90, 95 and $100 \%$ ) and then embedded into paraffin blocks. A total of 5 blocks were selected at random and each one was cut into three sections on a paraffin microtome with a thickness of 4- $\mu \mathrm{m}$. Sections were deparaffinaged in dimethylbenzene, embedded in xylene I for $20 \mathrm{~min}$, xylene II for $20 \mathrm{~min}$, absolute ethyl alcohol I for $10 \mathrm{~min}$, absolute ethyl alcohol II for $10 \mathrm{~min}, 95 \%$ alcohol for $5 \mathrm{~min}, 90 \%$ alcohol for $5 \mathrm{~min}, 80 \%$ alcohol for $5 \mathrm{~min}$ and $70 \%$ alcohol for $5 \mathrm{~min}$ successively prior to washing with water. Nuclei staining with hematoxylin: Sections were stained with hematoxylin for 3-8 min and eosin 1-3 min. The liver tissue structures were observed with a light microscope (magnification, x400; Nikou Corporation; Tokyo, Japan).

Blood from celiac vein was used to determine the biochemical indicators. A Hitachi 7060 automatic biochemical analyzer (Hitachi, Ltd., Tokyo, Japan) was used to detect serum alanine transaminase (ALT; Wako Pure Chemical Industries, Ltd., Osaka, Japan), aspartate transaminase (AST; Wako Pure Chemical Industries, Ltd., Osaka, Japan), total cholesterol (TC; Beijing Homa Biological Engineering Co., Ltd., Beijing, China), total triglycerides (TG; Beijing Homa Biological Engineering Co., Ltd.), high-density lipoprotein cholesterol (HDL-C; Medicalsystem Biotechnology Co., Ltd., Ningbo, Zhejiang), low-density lipoprotein cholesterol (LDL-C; Medicalsystem Biotechnology Co., Ltd.), fasting blood glucose (FBG; Autec Diagnostic; Baden-Württemberg, Germany). An abbott i2000 (Abbott Molecular Inc., Chicago, IL, USA) was used to detect serum fasting insulin (FINS; Abbott Molecular Inc.).
Determination of the activity of DPPIV by a Gly-Pro-7-amino-4-methylcoumarin (AMC) fluorescence method. The operations were conducted according to the manufacturer's instructions of a DPPIV kit (AAT Bioquest Inc., California, USA). A total of $5 \mathrm{mg}$ of Gly-Pro-AMC (a sensitive fluorogenic substrate; molecular weight, 443.37) was introduced into $563.5 \mu \mathrm{l}$ of dimethyl sulfoxide (DMSO) to prepare $20 \mathrm{mM}$ substrate DMSO stock solution. The substrate DMSO stock solution were diluted into $100 \mu \mathrm{M}$ substrate DMSO stock solution with $50 \mathrm{mM}$ Tris- $\mathrm{HCl}$, and mixed with rat serum $(150 \mu \mathrm{l}: 150 \mu \mathrm{l})$ for $1 \mathrm{~h}$ at room temperature. The fluorescence intensity was measured with an enzyme-labeled instrument (Biotek Corporation; Broadview, IL, USA) at $\mathrm{Ex} / \mathrm{Em}=380 / 500 \mathrm{~nm}$. The activity inhibition rates of DPPIV were calculated with the following equation (16): Activity inhibition rate of DPPIV $=$ (fluorescence intensity of negative control group - fluorescence intensity of each administration group) / fluorescence intensity of negative control group x $100 \%$.

Detection of DPPIV protein expression by an immunohistochemical staining method. Liver tissue were fixed with formaldehyde and hydrated with gradient ethanol as described in HE staining. Following citrate buffer antigen retrieval, slices were incubated with $3 \%$ deionized water for $20 \mathrm{~min}$ and then washed with PBS for 5 min, 3 times. Following blocking with 5-10\% normal goat serum (Boster Biological Technology Co., Ltd.) for $25 \mathrm{~min}$ at room temperature, $4 \mu \mathrm{m}$ thick slices were incubated with DPPIV primary antibody (cat. no. 10940_1_ap; 1:100; Proteintech group, Inc., Wuhan, Hubei) at $4^{\circ} \mathrm{C}$ overnight. Subsequently, samples were incubated with goat anti-rabbit secondary antibody (cat. no. GB23303; 1:200; Wuhan Goodbio Technology Co., Ltd., Wuhan, Hubei) for $50 \mathrm{~min}$ at room temperature. Following color development with 3,3'-diaminobenzidine reagent for $5 \mathrm{~min}$ at room temperature, the sections were mounted and observed under a light microscope (magnification, x400; Nikou Corporation), and the appearance of a brown-yellow liver cell membrane indicated positive expression. A total of five non-overlapping fields of view were selected and the immunohistochemical scores (IHS) method (17) was used to score samples according to the percentage of positive cells and their staining intensity, and the results were analyzed with Image Pro-Plus version 6.0 (Media Cybernetics, Rockville, Maryland, USA).

Detection of the expression of DPPIV protein in liver tissues by western blot analysis. Total protein was extracted from the liver tissue. The total sample protein was extracted using the holoprotein extraction kit (Nanjing KeyGEN Biotech Co., Ltd., Nanjing, China). Liver tissue (100 mg) was placed in a petri dish and cut into blocks ( $3 \times 3 \mathrm{~mm}), 0.5-1 \mathrm{ml}$ of $0-4^{\circ} \mathrm{C}$ Lysis Buffer were added, which contained $5 \mu \mathrm{l}$ of phosphatase inhibitor, $1 \mu \mathrm{l}$ of protease inhibitor and $5 \mu 1100 \mathrm{mM}$ phenylmethylsulfonyl fluoride. The mixture was homogenized 15 times under $4^{\circ} \mathrm{C}$ prior to centrifugation at $10,625 \mathrm{x}$ g for $15 \mathrm{~min}$ at $4^{\circ} \mathrm{C}$. The supernatants were collected and protein quantification were performed using the BCA method: and the absorbance was read at $562 \mathrm{~nm}$ with a ultraviolet spectrophotometer. In total, $20 \mu \mathrm{g}$ protein was loaded into each well and subjected to $5 \%$ SDS-PAGE at $60 \mathrm{~mA}$. The proteins were then transferred onto a polyvinylidene difluoride membrane at $100 \mathrm{~V}$ constant 
voltage for $65 \mathrm{~min}$, and incubated using 5\% skimmed milk powder overnight at $4^{\circ} \mathrm{C}$. The PVDF membrane was incubated with DPPIV and $\beta$-actin primary antibody (1:500, Abcam; Cambridge, $\mathrm{UK}$ ) for $2 \mathrm{~h}$ at room temperature, and then washed three times for $10 \mathrm{~min}$ with phosphate buffered saline with Tween 20 (PBST). The PVDF membrane were incubated with horseradish peroxidase labeled goat anti-rabbit secondary antibody (cat. no. ab97200; 1:1,000; Abcam, Cambridge, England) for $1 \mathrm{~h}$ at room temperature and washed three times for $10 \mathrm{~min}$ with PBST. The diaminobenzidine developer were prepared before use. The washed PVDF membrane was put into the developer for $3 \mathrm{~min}$, and then the diaminobenzidine reaction was terminated with water. The membrane was imaged with a full-automatic digital gel image analysis system (Shanghai Tanon Technology Co., Ltd., Shanghai, China) and the results were analyzed with Image J version 1.48 (National Institutes of Health, Bethesda, USA).

Statistical methods. Data are presented as the mean \pm standard deviation. Comparison among groups was performed using one-way analysis of variance and the least significant difference method was used for pairwise comparison among groups when the variances were equal. In addition, the Games-Howell method was used for pairwise comparison among groups when the variances were not equal. SPSS version 18.0 software (SPSS, Inc., Chicago, IL, USA) was used for the statistical analysis. $\mathrm{P}<0.05$ was considered to indicate a statistically significant difference.

\section{Results}

Liver index results of young rats. Compared with the normal group, the liver index in the model group increased significantly $(\mathrm{P}<0.05)$. Compared with the model group, the liver index in the ACNAC groups were significantly decreased $(\mathrm{P}<0.05)$, and the most marked decrease was observed in the high-dose group. No significant difference was observed in liver index between the ACNAC high-dose and PPC groups (Fig. 1).

HE staining of liver tissue of young rats. In the normal group and under a light microscope the structure of liver tissues of young rats was clear and complete. Additionally, the structure of the liver lobule was normal and the liver cells were distributed around the central vein in a radioactive arrangement. In the model group, fatty degeneration in liver cells of young rats was markedly increased. Furthermore, diffuse hepatocellular fatty degeneration was accompanied with ballooning degeneration, and there were areas of spotty necrosis of liver cells accompanied with hepatic fibrosis around the central veins. Compared with the model group, in the ACNAC groups, particularly the high-dose group, fatty degeneration and the degree of ballooning degeneration of liver tissue of young rats was markedly alleviated, with no evident inflammatory cell infiltration or focal necrosis occurring in the central veins. Additionally, compared with the model group, the PPC group has no evident fatty degeneration and occasional microvesicular fat droplet vacuoles. Finally, there were no marked differences observed between the PPC and ACNAC high-dose groups (Fig. 2).
Serum biochemical indices in young rats. Compared with the model group, the high-dose ACNAC group had significantly reduced ALT, AST, TC, TG, LDL-C, FBG and FINS and increased HDL-C levels $(\mathrm{P}<0.05)$. Furthermore, no significant differences were observed in the TC, TG, LDL-C and HDL-C levels between the ACNAC high-dose and PPC groups (P>0.05; Figs. 3 and 4).

Determination of inhibition rate of DPPIV activity via the Gly-Pro-AMC fluorescence method. Serum was added to a fully functional enzyme standard instrument to detect the inhibitory effect of ACNAC on the activity of DPPIV. Compared with the normal group, the model group exhibited a significantly decreased inhibition rate of DPPIV activity of $-29.54 \pm 13.32 \%$ in the serum $(\mathrm{P}<0.05)$. Compared with the model group, the high-dose ACNAC group exhibited a significantly increased inhibition rate of DPPIV activity of $49.50 \pm 5.56 \%$ in the serum $(\mathrm{P}<0.05)$. In comparison with the PPC group, which had an inhibition rate of $46.42 \pm 5.88 \%$, the ACNAC high-dose group had an increased inhibition rate of DPPIV activity in the serum and the intergroup differences; however, this was not statistically significant ( $P>0.05$; Fig. 1).

Detection of DPPIV protein expression by immunohistochemical staining. Positive expression of DPPIV is indicated by a brown-yellow liver cell membrane. Compared with the percentage of DPPIV-positive cells of the liver cell membrane in the normal group $(89.19 \pm 6.23 \%)$ the expression of positive cells in the model group $(43.48 \pm 4.99 \%)$ was significantly decreased $(\mathrm{P}<0.05)$. Compared with the model group, a significantly higher percentage of DPPIV-positive cells were detected in the liver cell membranes of the ACNAC groups $(\mathrm{P}<0.05)$, and the positive expression of DPPIV increased with the decrease of fatty degeneration of liver tissue, particularly in the high-dose ACNAC group in which the percentage $(68.57 \pm 6.48 \%)$ of positive cells was most markedly increased. In comparison with the PPC group $(67.90 \pm 6.45 \%)$, the percentage of positive cells of DPPIV-positive cells in the ACNAC high-dose group was slightly increased; however there was no statistical significant (P>0.05; Figs. 1 and 5).

Results of DPPIV protein expression detection by western blot analysis. In comparison with the normal group, the DPPIV protein expression in the model group was significantly decreased $(\mathrm{P}<0.05)$. Compared with the model group, DPPIV protein expression in the low-, medium- and high-dose ACNAC groups significantly increased in a dose-dependent manner $(\mathrm{P}<0.05$; Fig. 6).

\section{Discussion}

NAFLD has become the most common liver disease influencing the health of children and adolescents (18-20). According to a previous study, NAFLD patients most likely account for $30 \%$ among 70 million individuals in the USA and up to $8 \%$ among children and adolescents between 2 and 19 years old (20). The pathogenesis in children may be different from adults; however, the overall incidence trend is simple fatty liver, fatty liver hepatitis, liver fibrosis and even liver cirrhosis (21). Consequently, it is necessary to screen 
A

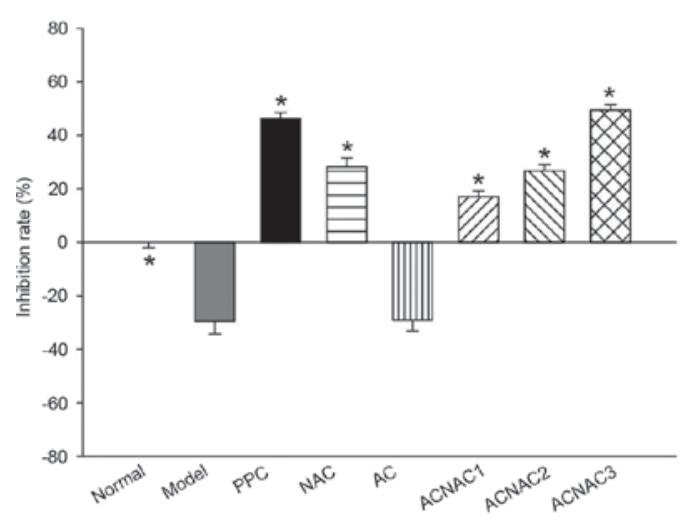

B

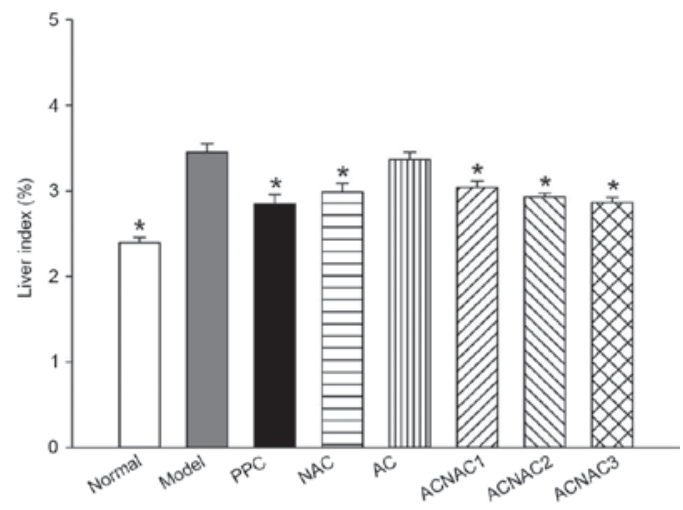

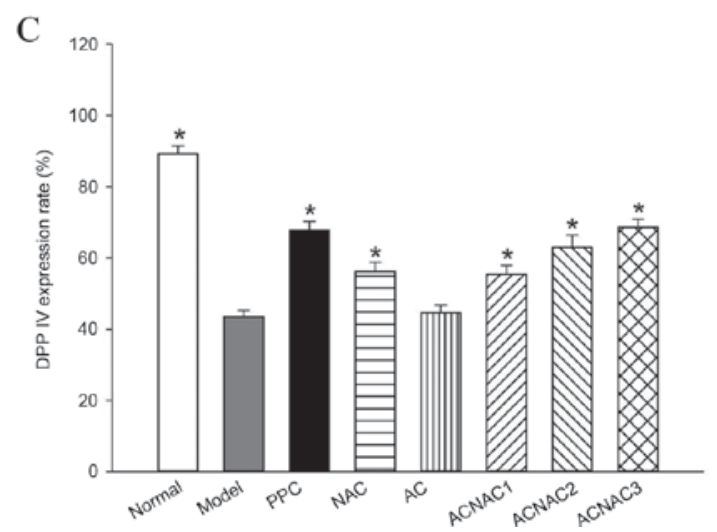

Figure 1. Comparative results of liver index, serum DPPIV activity inhibition ratio and DPPIV expression rate in the young rats of each group. (A) Liver index, (B) DPPIV inhibition and (C) DPPIV expression rates. Bars represent the mean \pm standard deviation of results obtained per experimental group. ${ }^{*} \mathrm{P}<0.05$ vs. the model group determined by one-way analysis of variance and the least significant difference method. Normal, normal group; model, model group; PPC, polyene phosphatidyl choline group; NAC, $N$-acetylcysteine control group; AC, activated carbon release microcapsule control group; ACNAC1, $N$-acetylcysteine activated carbon release microcapsule low-dose group; ACNAC2, $N$-acetylcysteine activated carbon release microcapsule middle-dose group; ACNAC3, $\mathrm{N}$-acetylcysteine activated carbon release microcapsule high-dose group; DPPIV, dipeptidyl peptidase IV.

the children with such a disease risk as soon as possible and to study the pathogenesis $(22,23)$. For obese children with NAFLD, any unhealthy dietary and lifestyle habits should be corrected (24-26), aerobic exercise encouraged, and their weight should be controlled to reduce insulin resistance so that liver damage may be reversed at an early stage (27-29). If necessary, drug intervention with low levels of toxicity and side effects, and evident curative effects may be used to effectively reduce the occurrence of NAFLD and other chronic metabolic diseases in children $(30,31)$.

At present, the main issues facing medication for children are imperfect drug safety information and the lack of medicinal varieties (32), specification and special formulations, which results in adultification of medicine in children and various disadvantages (33). In order to solve these problems, China has increased the input of oral sustained-release therapies, controlled release preparations, orally disintegrating tablets and transdermal absorption preparations, with the aim of developing preparations that conform to the characteristics of medication in infants and children and that have a low toxicity, few side effects and a high bioavailability (34). For the present study, the advantage of adsorptive behavior and in vitro release properties of activated carbon for medicine were considered, and activated carbon NAC sustained-release microcapsules were studied in order to improve in oxidizability and bioavailability of NAC, reduce the dose in clinical use, increase drug stability and elucidate the ideal pharmaceutical preparation for infants and children.

DPPIV is widely found in organisms and its expression is predominantly in epithelial cells, lymphocytes, endothelial cells and fibroblasts. Previous studies have demonstrated that DPPIV has a multiple-effect on biological activity $(35,36)$, particularly the protease in known DPPIV affects fat metabolism, predominantly lipid metabolism of an organism through neuropeptide $\mathrm{Y}$ and inactivation of other polypeptides.

The present study used a high-fat diet to induce a model of young rats with NAFLD and combines children's dietary structure and habits in order to establish a young animal model with abdominal obesity, lipid metabolism disorder and elevated liver enzyme levels that are similar to children with NAFLD. The results demonstrated that compared with the normal group, the model group exhibited weight gain, liver index increase, visceral fat deposition, evident liver volume increase, and the livers were grayish-yellow color and had a greasy surface area in young rats. Additionally, different degrees of fatty changes and ballooning degeneration were observed via HE pathological staining. In addition, vacuoles of different sizes were filled in the cells, and various areas were accompanied by inflammatory cell infiltration and occasional focal necrosis. Furthermore, the activity of ALT, AST, TC, TG, LDL-C, FBG, FINS and DPPIV in the serum was evidently increased and the expression of HDL-C and 

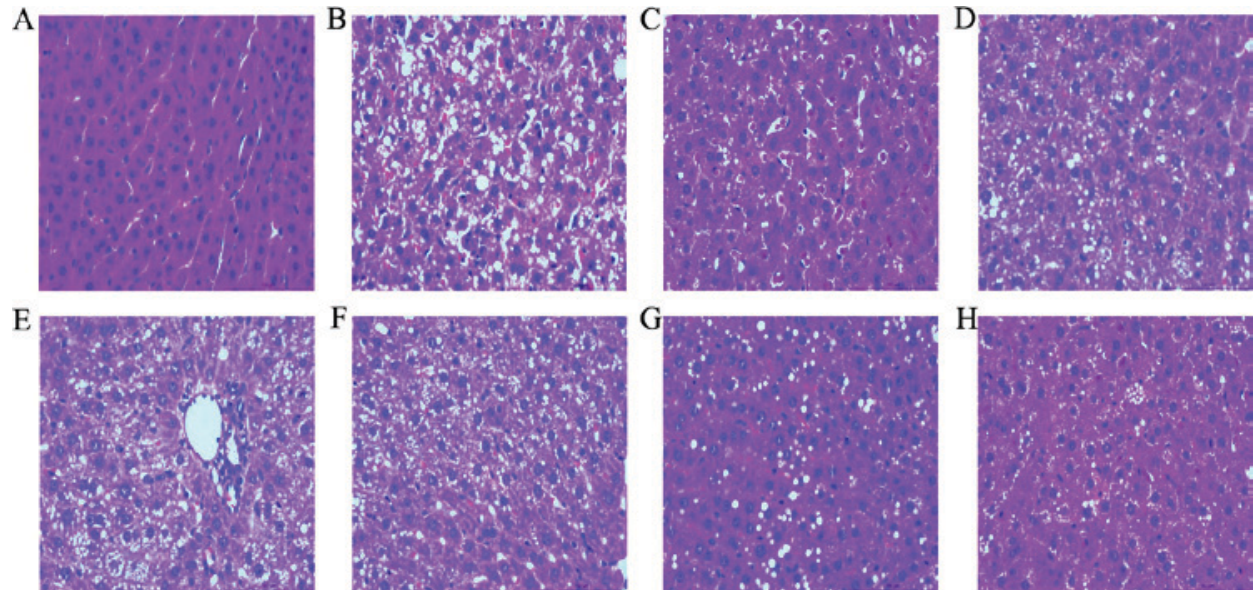

Figure 2. Effect of activated carbon release microcapsule on liver fatty degeneration in young rats with non-alcoholic fatty liver disease. Pathological test results of liver tissue of young rats with hematoxylin and eosin staining (magnification, x400) in each group. (A) Normal, (B) model, (C) polyene phosphatidyl choline, (D) $N$-acetylcysteine control, (E) activated carbon release microcapsule control, (F) $N$-acetylcysteine activated carbon release microcapsule low-dose, (G) $\mathrm{N}$-acetylcysteine activated carbon release microcapsule middle-dose and (H) $\mathrm{N}$-acetylcysteine activated carbon release microcapsule high-dose groups.

A

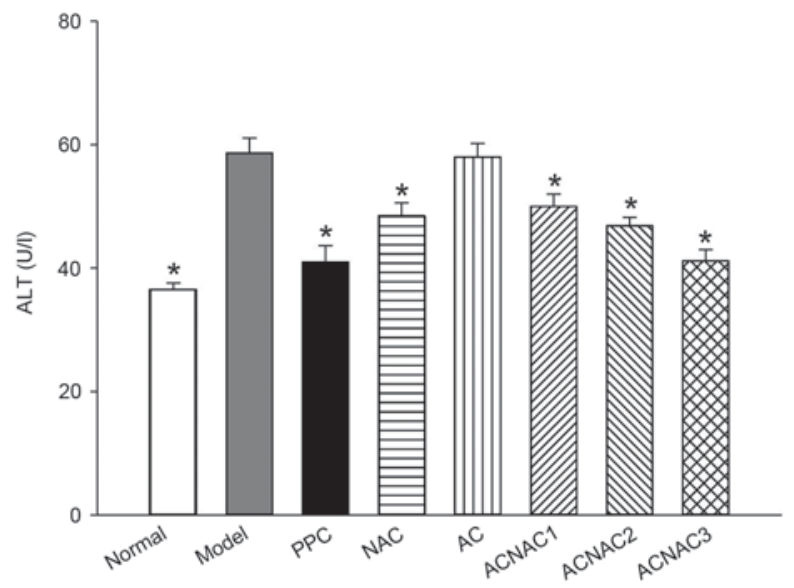

$\mathrm{C}$

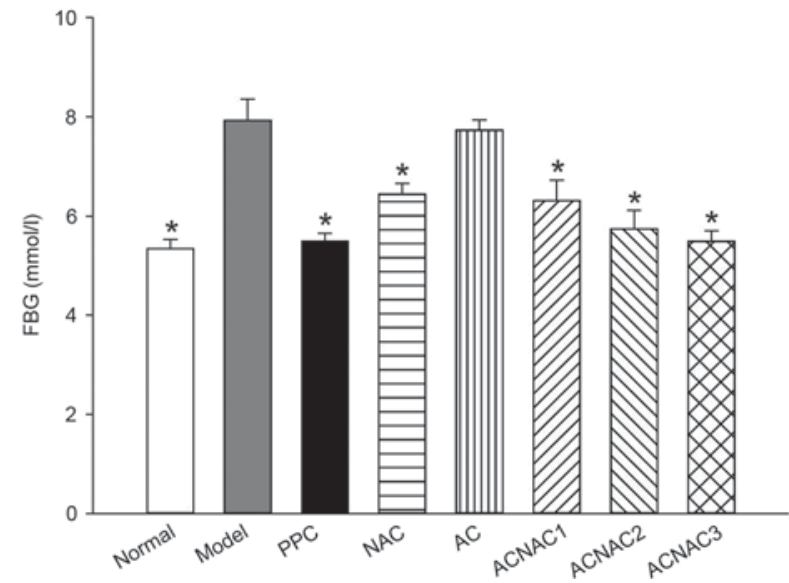

B

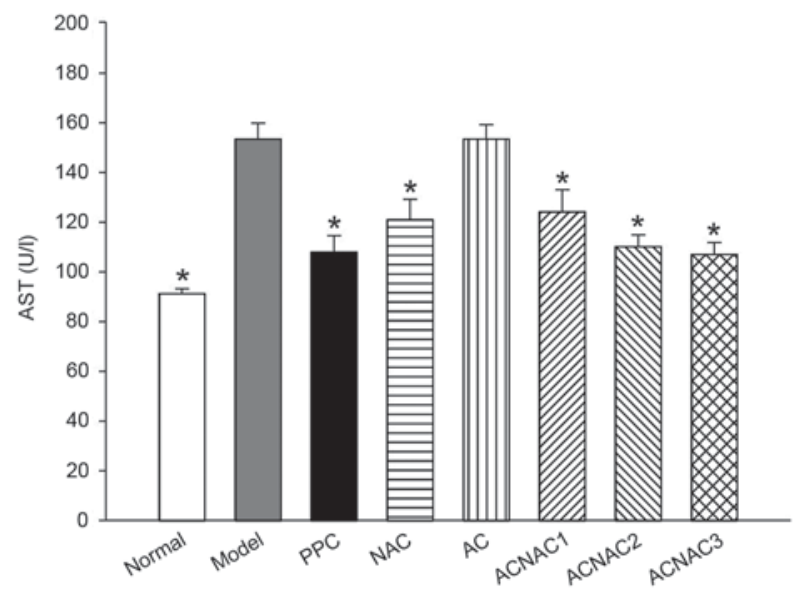

$\mathrm{D}$

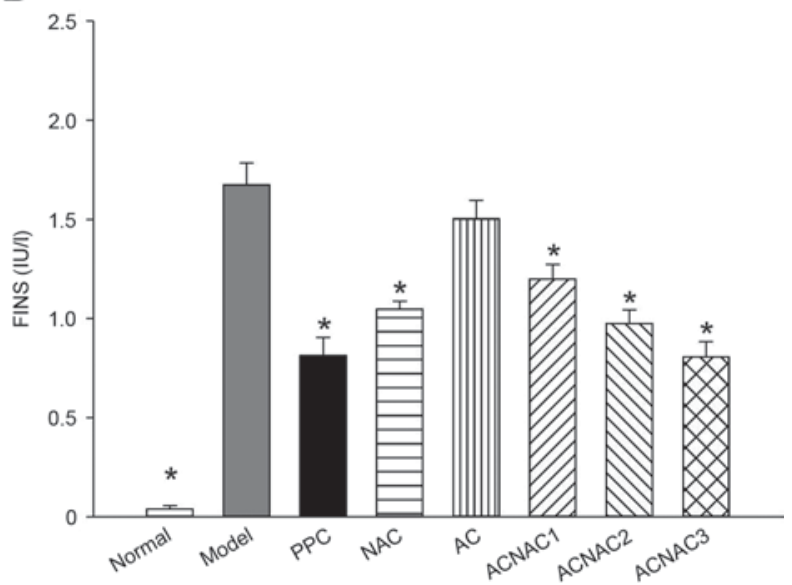

Figure 3. Comparative results of ALT, AST, FBG and FINS serums in young rats of each group. (A) ALT, (B) AST, (C) FBG and (D) FINS. Bars represent the mean \pm standard deviation of the results obtained per experimental group. ${ }^{*} \mathrm{P}<0.05$ vs. the model group, as determined by one-way analysis of variance and the least significant difference method. Normal, normal group; model, model group; PPC, polyene phosphatidyl choline group; NAC, $N$-acetylcysteine control group; AC, activated carbon release microcapsule control group; ACNAC1, $\mathrm{N}$-acetylcysteine activated carbon release microcapsule low-dose group; ACNAC2, $N$-acetylcysteine activated carbon release microcapsule middle-dose group; ACNAC3, $N$-acetylcysteine activated carbon release microcapsule high-dose group; ALT, alanine transaminase; AST, aspartate transaminase; FBG, fasting blood glucose; FINS, fasting insulin.

DPPIV protein in the liver cell membrane were markedly decreased.
A previous study by Balaban et al (37) demonstrated that the activity of DPPIV in the serum in patients with 

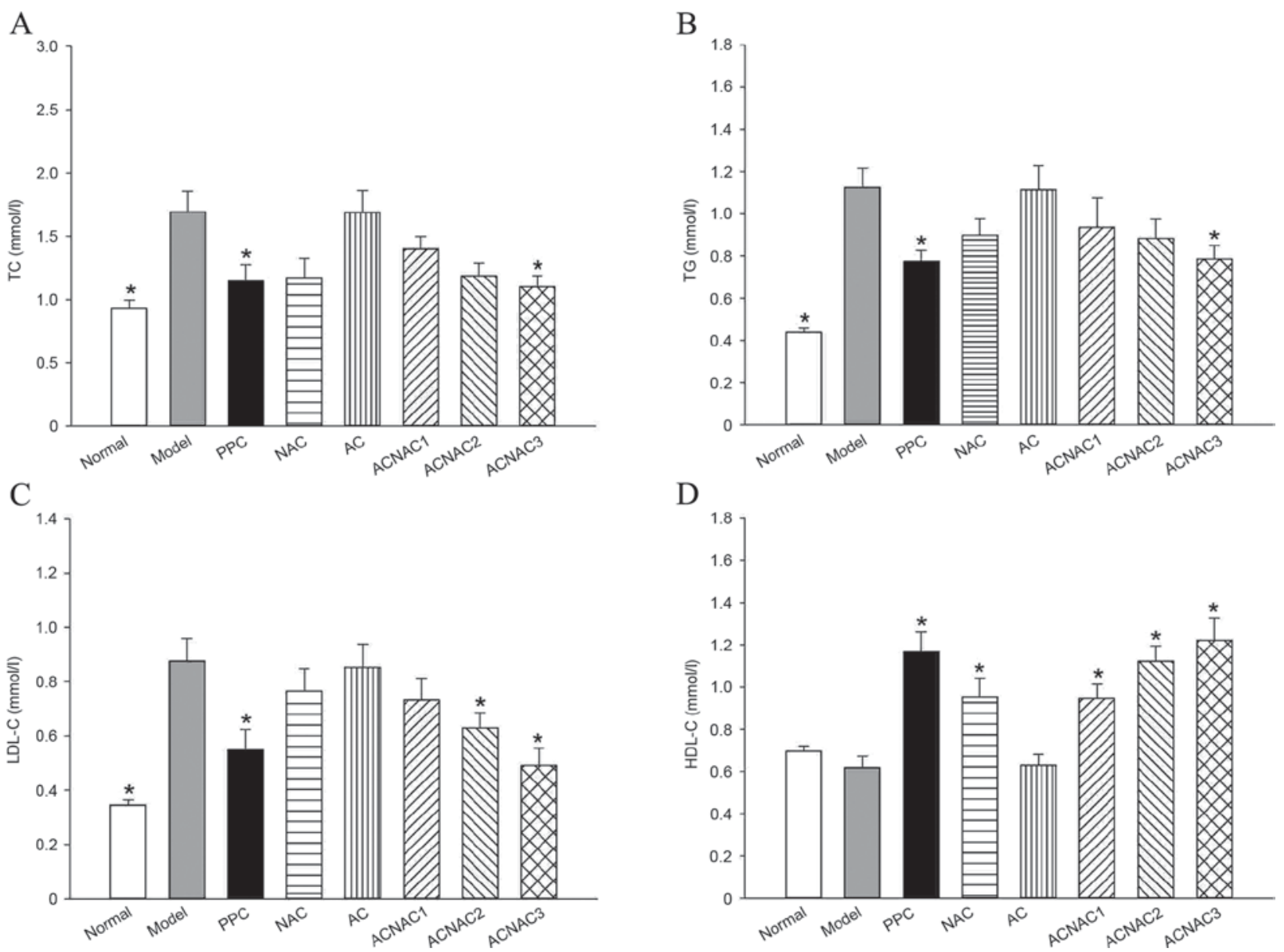

Figure 4. Comparative results of TC, TG, HDL-C and LDL-C serums in the young rats of each group. (A) TC, (B) TG, (C) LDL-C and (D) HDL-C. Bars represent the mean \pm standard deviation of the results obtained per experimental group. $\mathrm{P}<0.05$ vs. the model group, as determined by one-way analysis of variance and least significant difference method. Normal, normal group; Model, model group; PPC, polyene phosphatidyl choline group; NAC, $N$-acetylcysteine control group; AC, activated carbon release microcapsule control group; ACNAC1, $N$-acetylcysteine activated carbon release microcapsule low-dose group; ACNAC2, $\mathrm{N}$-acetylcysteine activated carbon release microcapsule middle-dose group; ACNAC3, $\mathrm{N}$-acetylcysteine activated carbon release microcapsule high-dose group; TC, total cholesterol; TG, total triglycerides; LDL-C, low density lipoproteins cholesterol; HDL-C, high density lipoproteins cholesterol.
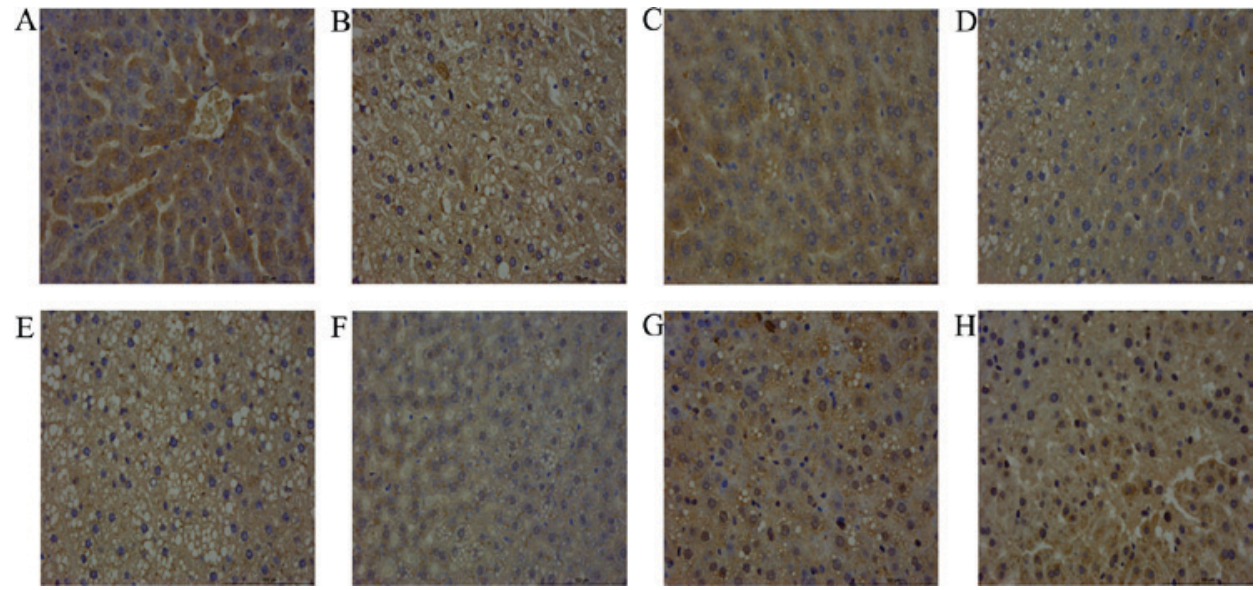

Figure 5. Immunohistochemical detection of dipeptidyl peptidase IV in liver tissues of young rats in each group. Representative images of immunohistostaining of DPPIV that was indicated with brownish yellow stain (magnification, x400). (A) Normal, (B) model, (C) polyene phosphatidyl choline, (D) $N$-acetylcysteine control, (E) activated carbon release microcapsule control, (F) $N$-acetylcysteine activated carbon release microcapsule low-dose, (G) $N$-acetylcysteine activated carbon release microcapsule middle dose and $(\mathrm{H}) \mathrm{N}$-acetylcysteine activated carbon release microcapsule high-dose groups.

non-alcoholic steatohepatitis was markedly higher than those in the normal group. Furthermore, the degree of pathological change of liver tissue and degree of fat change of liver cells of NAFLD were associated with the serum DPPIV activity and the intensity of protein expression. It is thought that DPPIV may be closely associated with the pathogenesis of 

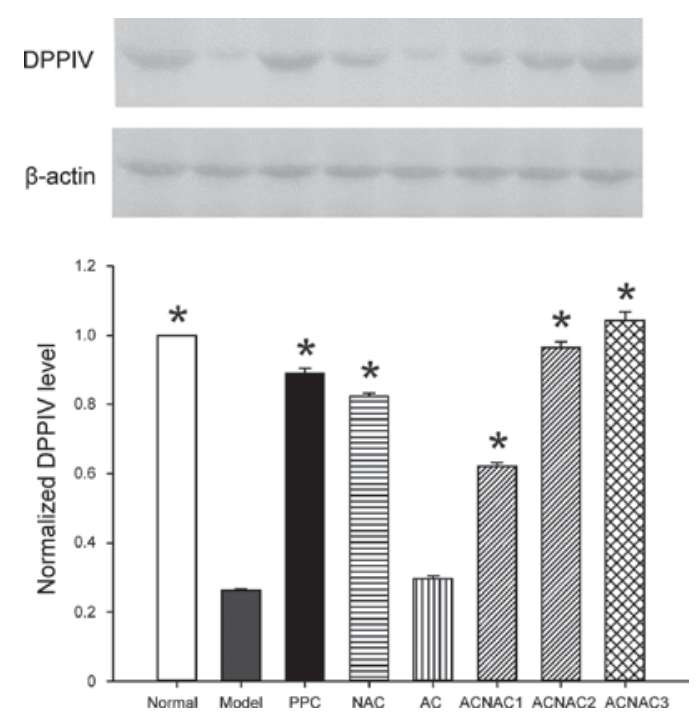

Figure 6. Results of DPPIV protein expression detection via western blot analysis. ${ }^{*} \mathrm{P}<0.05$ vs. the model group. Normal, normal group; model, model group; PPC, polyene phosphatidyl choline group; NAC, $N$-acetylcysteine control group; AC, activated carbon release microcapsule control group ACNAC1, $N$-acetylcysteine activated carbon release microcapsule low-dose; ACNAC2, $N$-acetylcysteine activated carbon release microcapsule middle-dose and ACNAC3, $N$-acetylcysteine activated carbon release microcapsule high dose groups; DPPIV, dipeptidyl peptidase IV.

NAFLD (38). It has previously been revealed that the DPPIV inhibitor may improve fatty degeneration of the liver in rats (39). Furthermore, a previous clinical experiment demonstrated that the DPPIV inhibitor may also improve transaminase levels and ballooning degeneration of liver cells in patients with NAFLD (40). The present experimental results demonstrated that, compared with the model group, the ACNAC groups (particularly the high-dose group) exhibited different degrees of improvement in the indexes detailed above, which reduced the activity of ALT, AST and DPPIV and the content of TC, TG, LDL-C, FBG and FINS, increased the content of HDL-C and strengthened DPPIV protein expression in the liver cell membrane. This is consistent with the results of the study performed by Balaban et al (37). These findings suggest that ACNAC may have the effect of a DPPIV inhibitor in the treatment of NAFLD in young rats, which provides novel rationale for the use of NAC in studies of NAFLD/non-alcoholic steatohepatitis in young rats, and provides theoretical knowledge for ideal pharmaceutical preparations for infants and children. However, the underlying mechanism still remains to be investigated in future experiments.

\section{Acknowledgements}

The present study was supported by Hangzhou Science and Technology Development projects (grant nos. 20130633B09, 20140633B29 and 20142013A60).

\section{References}

1. Roth CL, Elfers CT, Figlewicz DP, Melhorn SJ, Morton GJ, Hoofnagle A, Yeh MM, Nelson JE and Kowdley KV: Vitamin $\mathrm{D}$ deficiency in obese rats exacerbates nonalcoholic fatty liver disease and increases hepatic resistin and Toll-like receptor activation. Hepatology 55: 1103-1111, 2012.
2. Fan JG: Epidemiology of alcoholic and nonalcoholic fatty liver disease in China. J Gastroenterol Hepatol 28 (Suppl 1): S11-S17, 2013.

3. Fan JG and Zeng MD: Fatty liver disease. Version 2, Beijing: People's Medical Publishing House 20, 2013.

4. Farrell GC, Mc Cullough AJ and Day CP (eds): Non-Alcoholic Fatty Liver Disease: A Practical Guide. Wiley-Blackwell, Oxford, p216, 2013.

5. Loomba R, Sirlin CB, Schwimmer JB and Lavine JE: Advances in pediatric nonalcoholic fatty liver disease. Hepatology 50: 1282-1293, 2009.

6. Gorrell MD: Dipeptidyl peptidase IV and related enzymes in cell biology and liver disorders. Clin Sci (Lond) 108: 277-292, 2005.

7. Fimeisz G, Varga T, Lengyel G, Fehér J, Ghyczy D, Wichmann B Selmeci L, Tulassay Z, Rácz K and Somogyi A: Seram dipeptidyl peptidase-4 activity in insulin resistant patients with nonalcoholic fatty liver disease: A novel liver disease biomarker. PLoS One 5: e12226, 2010.

8. Ragab D, Laird M, Duffy D, Casrouge A, Mamdouh R, Abass A Shenawy DE, Shebl AM,Elkashef WF,Zalata KR, et al: CXCL10 antagonism and plasma sDPPIV correlate with increasing liver disease in chronin HCV genotype 4 infected patients. Cytokine 63: 105-112, 2013.

9. Ottobelli Chielle E, de Souza WM, da Silva TP, Moresco RN and Moretto MB: Adipocytokines, inflammatory and oxidative stress markers of clinical relevance altered in young overweight/obese subjects. Clin Biochem 49: 548-553, 2016.

10. Machado MV, Kruger L, Jewell ML, Michelotti GA, Pereira Tde A, Xie G, Moylan CA and Diehl AM: Vitamin B5 and $\mathrm{N}$-acetylcysteine in nonalcoholic steatohepatitis: A preclinical study in a dietary mouse model. Dig Dis Sci 61: 137-148, 2016.

11. Qu QL, Zhang YG, Yang LZ and Sun L: Intraperitoneal chemotherapy with mitomycin $\mathrm{C}$ bound to activated carbon nanoparticles for nude mice bearing human gastric carcinoma. Zhonghua Zhong Liu Za Zhi 28: 257-260, 2006 (In Chinese).

12. Wang FG, Zhuang RX, Xi JJ, Fang HY and Gao JQ: Preparation and distribution in mice of acetylcysteine nanoparticles. Zhongguo Yiyao Gongye Zazhi 43: 572-576, 2012 (In Chinese).

13. Wang FG, Shen YQ, Zhuang RX, Xi JJ and Fang HY: Study on preparation of acetylcysteine nanoparticles. Strait Pharmaceut J 25: 15-17, 2013.

14. Jia L, Wang J, Shi JP, Wang F, Gang H, Zhu M and Lou G: The effect of $\mathrm{N}$-acetylcysteine nano-carbon on anti-oxidative capacity in non-alcoholic steatohepatitis rats. Zhonghua Shi Yan He Lin Chuang Bing Du Xue Za Zhi 28: 4-6, 2014 (In Chinese).

15. Fang HY, Zhang RX, Xi JJ, Sun JJ, Shao YD, Si ZZ, Pan XW and Wang FG: Role of treatment on liver fibrosis of activated carbon N-acetylcysteine microcapsule. Zhong Guo Lin Chuang Yao Li Xue Yu Zhi Liao Xue Bian Ji Bu 20: 976-980, 2015 (In Chinese).

16. Pei Z, Li X, Longenecker K, von Geldern TW, Wiedeman PE, Lubben TH, Zinker BA, Stewart K, Ballaron SJ, Stashko MA, et al: Discovery, structure-activity relationship, and pharmacological evaluation of (5-substituted-pyrrolidinyl-2-carbonyl)-2-cyanopyrrolidines as potent dipeptidyl peptidase IV Inhibitors. J Med Chem 49: 3520-3535, 2006.

17. Soslow RA, Dannenberg AJ, Rush D, Woerner BM, Khan KN, Masferrer J and Koki AT: COX-2 is expressed in human pulmonary, colonic, and mammary tumors. Cancer 89: 2637-2645, 2000.

18. Lavine JE and Schwimmer JB: Nonalcoholic fatty liver disease in the pediatric population. Clin Liver Dis 8: 549-558, viii-ix, 2004.

19. Fraser A, Longnecker MP and Lawlor DA: Prevalence of elevated alanine aminotrasferase among US adolescents and associated factors: NHANES 1999-2004. Gastroenterology 133: 1814-1820, 2007.

20. Schwimmer JB, Deutsch R, Kahen T, Lavine JE, Stanley C and Behling C: Prevalence of fatty liver in children and adolescents. Pediatric 118: 1388-1393, 2006.

21. Kohli R, Boyd T, Lake K, Dietrich K, Nicholas L, Balistreri WF, Ebach D, Shashidhar H and Xanthakos SA: Rapid progression of NASH in childhood. J Pediatr Gastroenterol Nutr 50: 453-456, 2010.

22. Holterman A, Gurria J, Tanpure S and DiSomma N: Nonalcoholic fatty liver disease and bariatric surgery in adolescents. Semin Pediatr Surg 23: 49-57, 2014.

23. Alterio A, Alisi A, Liccardo D and Nobili V: Non-alcoholic fatty liver and metabolic syndrome in children: A vicious circle. Horm Res Paediatr 82: 283-289, 2014.

24. Ozhan B, Ersoy B, Kiremitci S, Ozkol M and Taneli F: Insulin sensitivity indices: Fasting versus glucose-stimulated indices in pediatric non-alcoholic fatty liver disease. Eur Rev Med Pharmacol Sci 19: 3450-3458, 2015. 
25. Anderson EL, Howe LD, Jones HE, Higgins JP, Lawlor DA and Fraser A: The prevalence of non-alcoholic fatty liver disease in children and adolescents: A systematic review and meta-analysis. PLoS One 10: e0140908, 2015.

26. Jogo R, Ness AR, Emmett P, Mattocks C, Jones L and Riddoch CJ: Obesogenic diet and physical activity: Independent or associated behaviours in adolescents? Public Health Nutr 13: 673-681, 2010.

27. Monteiro PA, Antunes Bde M, Silveira LS, Christofaro DG, Fernandes RA and Freitas Junior IF: Body composition variables as predictors of NAFLD by ultrasound in obese children and adolescents. BMC Pediatr 14: 25, 2014

28. Manco M, Bedogni G, Marcellini M, Devito R, Ciampalini P, Sartorelli MR, Comparcola D, Piemonte F and Nobili V: Waist circumference correlates with liver fibrosis in children with non-alcoholic steatohepatitis. Gut 57: 1283-1287, 2008.

29. Schwimmer JB, Celedon MA, Lavine JE, Salem R, Campbell N, Schork NJ, Shiehmorteza M, Yokoo T, Chavez A, Middleton MS and Sirlin CB: Heritability of nonalcoholic fatty liver disease. Gastroenterology 136: 1585-1592, 2009.

30. Alisi A and Nobili V: Non-alcoholic fatty liver disease in children now: Lifestyle changes and pharmacologic treatments. Nutrition 28: 722-726, 2012.

31. Black LJ, Jacoby P, She Ping-Delfos WC, Mori TA, Beilin LJ, Olynyk JK, Ayonrinde OT, Huang RC, Holt PG, Hart PH, et al: Low serum 25-hydroxyvitamin D concentrations associate with non-alcoholic fatty liver disease in adolescents independent of adiposity. J Gastroenterol Hepatol 29: 1215-1222, 2014.
32. Abdel-Rahman SM, Amidon GL, Kaul A, Lukacova V, Vinks AA and Knipp GT: Summary of the national institute of child health and human development-best pharmaceuticals for children act pediatric formulation initiatives workshop-pediatric biopharmaceutics classification system working group. Clin Ther 11: S11-S24, 2012.

33. Ma LX, Liu X, Yan GQ, et al: Problems and thoughts on children drug use. Chin Hospitals 16: 45-46, 2012.

34. Hu N: Present situation and development strategy of paediatric formulations. Med Sci 1: 239, 2015.

35. Drucker DJ and Nauck MA: The incretin system: Glucagons-like peptide-1 receptor agonists and dipeptidyl peptidase-4 inhibitors in type 2 diahetes. Lancet 368: 1696-1705, 2006.

36. Holst JJ: Glucagon-like peptide-1: From extract to agent. The claude bernard lecture, 2005. Diabetologia 49: 253-260, 2006.

37. Balaban YH, Korkusuz P, Simsek H, Gokcan H, Gedikoglu G, Pinar A, Hascelik G, Asan E, Hamaloglu E and Tatar G: Dipeptidyl peptidaseIV (DPPIV) in NASH patients. Ann Hepatol 6: 242-250, 2007.

38. Han Q: The study of the relationship between DPPIV and fatty liver disease inobese children and young rats. Tianjin Med Uni 5 . 2011.

39. Shirakawa J, Fujii H, Ohnuma K, Sato K, Ito Y, Kaji M, Sakamoto E, Koganei M, Sasaki H, Nagashima Y, et al: Diet-induced adipose tissue inflammation and liver steatosis are prevented by DPP-4 inhibition in diabetic mice. Diabetes 60: 1246-1257, 2011.

40. Yilmaz Y, Yonal O, Deyneli O, Celikel CA, Kalayci C and Duman DG: Effects of sitagliptin in diabetic patients with nonalcoholic steatohepatitis. Acta Gastroenterol Belg 75: 240-244, 2012. 